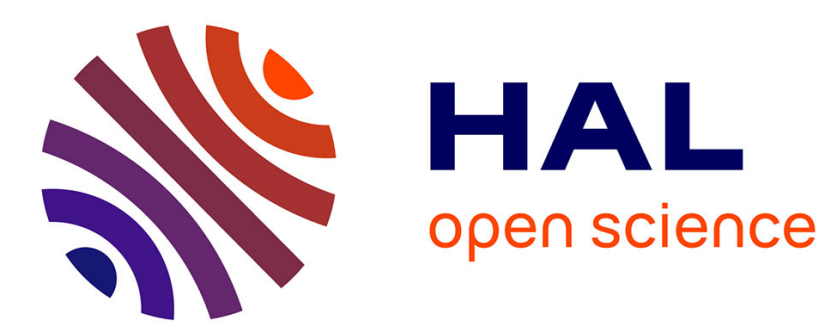

\title{
Existence of minimizers for the pure displacement problem in nonlinear elasticity
}

Cristinel Mardare

\section{To cite this version:}

Cristinel Mardare. Existence of minimizers for the pure displacement problem in nonlinear elasticity.

"Alexandru Myller" Mathematical Seminar, 2010, Romania. pp.181-190. hal-00959067

\section{HAL Id: hal-00959067 \\ https: / hal.sorbonne-universite.fr/hal-00959067}

Submitted on 13 Mar 2014

HAL is a multi-disciplinary open access archive for the deposit and dissemination of scientific research documents, whether they are published or not. The documents may come from teaching and research institutions in France or abroad, or from public or private research centers.
L'archive ouverte pluridisciplinaire HAL, est destinée au dépôt et à la diffusion de documents scientifiques de niveau recherche, publiés ou non, émanant des établissements d'enseignement et de recherche français ou étrangers, des laboratoires publics ou privés. 


\title{
Existence of minimizers for the pure displacement problem in nonlinear elasticity
}

\author{
Cristinel Mardare \\ Université Pierre et Marie Curie - Paris 6, Laboratoire Jacques-Louis Lions, \\ Paris, F-75005 France
}

\begin{abstract}
We show that the total energy of the pure displacement problem in nonlinear elasticity possesses a unique global minimizer for a large class of hyperelastic materials, including that of Saint Venant - Kirchhoff, provided the density of the applied forces are small in $L^{p}$-norm. We also establish a nonlinear Korn inequality with boundary showing that the $H^{1}$-distance between two deformation fields is bounded, up to a multiplicative constant, by the $L^{2}$-distance between their Cauchy-Green strain tensors.
\end{abstract}

\section{Introduction}

The total energy of a hyperelastic body undergoing a deformation $\mathbf{u}: \Omega \rightarrow \mathbb{R}^{3}$ from its reference configuration $\Omega \subset \mathbb{R}^{3}$ is

$$
J(\mathbf{u})=\int_{\Omega} \hat{W}(x, \nabla \mathbf{u}(x)) d x-\int_{\Omega} \mathbf{f}(x) \cdot \mathbf{u}(x) d x,
$$

where $\hat{W}: \bar{\Omega} \times \mathbb{M}_{+}^{3} \rightarrow \mathbb{R}$ is the stored energy function of the hyperelastic material constituting the body and $\mathbf{f}: \Omega \rightarrow \mathbb{R}^{3}$ is the density of the loads acting on the body, assumed here to be dead loads. The notations $\mathbb{M}_{+}^{3}$ and $\mathbf{f}(x) \cdot \mathbf{u}(x)$ designate respectively the set of all square matrices of order three with positive determinant and the inner product in $\mathbb{R}^{3}$.

If $\hat{W}$ is polyconvex and satisfies suitably growth conditions, then the total energy possesses a minimizer in an appropriate set of admissible deformations; cf. Ball [1]. If in addition the Euler-Lagrange equation corresponding to this minimization problem has a solution by the implicit function theorem, then this solution coincides with the minimizer above; cf. Zhang [8].

We show here that the total energy possesses a minimizer for some hyperelastic materials that does not meet the assumptions of Ball and Zhang. We first assume that $\hat{W}$ satisfies the axiom of material frame-indifference, so that there exists a function $W: \bar{\Omega} \times \mathbb{S}^{3}$ such that, for almost all $x \in \Omega$ and all $F \in \mathbb{M}_{+}^{3}$,

$$
\hat{W}(x, F)=W(x, E), E=\frac{1}{2}\left(F^{T} F-I\right) .
$$


The notations $\mathbb{S}^{3}$ and $I$ denote respectively the set of all symmetric matrices of order three and the identity matrix of order three. Then we assume that $W$ is of class $\mathcal{C}^{3}$ and satisfies the following property: $\frac{\partial W}{\partial E}(x, 0)=0$ for all $x \in \bar{\Omega}$ and there exists constants $\alpha>0$ and $\varepsilon>0$ such that, for all $x \in \bar{\Omega}, H \in \mathbb{S}^{3}, E \in \mathbb{S}^{3}$, $|E|<\varepsilon$,

$$
W(x, E+H) \geq W(x, E)+\frac{\partial W}{\partial E}(x, E): H+\alpha|H|^{2} .
$$

The notations $|H|, \Sigma: H$, and $\frac{\partial W}{\partial E}$ denote respectively the Frobenius norm of the matrix $H$, the inner product of the matrices $\Sigma$ and $H$, and the derivative of the function $W$ with respect to its second variable. The typical function $W$ satisfying our assumptions is the stored energy function of a Saint Venant Kirchhoff material, namely

$$
W(x, E)=\frac{\lambda}{2}(\operatorname{tr} E)^{2}+\mu|E|^{2},
$$

where $\lambda, \mu$ denote the Lamé's constants of the material and $\operatorname{tr} E$ denotes the trace of the matrix $E$.

Under these assumptions, we show that the total energy $J$ possesses a unique global minimizer in the set

$$
\mathcal{M}=\left\{\mathbf{u} \in W^{1,4}\left(\Omega ; \mathbb{R}^{3}\right) ; \operatorname{det}(\nabla \mathbf{u})>0 \text { a.e. in } \Omega, \mathbf{u}(x)=x \text { for all } x \in \partial \Omega\right\},
$$

provided that the density of the applied forces satisfies $\|\mathbf{f}\|_{L^{p}(\Omega)}<\delta$ for certain constants $p>3$ and $\delta>0$. This will be done by applying the implicit function theorem to the Euler-Lagrange equation derived from the total energy $J$, then applying Zhang's method to show that the solution $\mathbf{v}$ found by the implicit function theorem minimizes the functional $J$ over $\mathcal{M}$. The key of the proof is a new nonlinear Korn inequality which asserts that there exists a constant $C$ such that, for all $\mathbf{u} \in \mathcal{M}$,

$$
\|\nabla \mathbf{u}-\nabla \mathbf{v}\|_{L^{2}(\Omega)} \leq C\left\|\nabla \mathbf{u}^{T} \nabla \mathbf{u}-\nabla \mathbf{v}^{T} \nabla \mathbf{v}\right\|_{L^{2}(\Omega)} .
$$

This inequality is reminiscent of another nonlinear Korn inequality, established earlier by Ciarlet \& Mardare [4], showing that

$$
\inf _{R \in \mathbb{O}_{+}^{3}}\|\nabla \mathbf{u}-R \nabla \mathbf{v}\|_{L^{2}(\Omega)} \leq C(\mathbf{v})\left\|\nabla \mathbf{u}^{T} \nabla \mathbf{u}-\nabla \mathbf{v}^{T} \nabla \mathbf{v}\right\|_{L^{1}(\Omega)}^{1 / 2}
$$

for all $\mathbf{u} \in H^{1}\left(\Omega ; \mathbb{R}^{3}\right)$ satisfying $\operatorname{det} \nabla \mathbf{u}>0$ a.e. in $\Omega$. The notation $\mathbb{O}_{+}^{3}$ denotes the special orthogonal group of degree 3 . Thus we have been able to drop the exponent $1 / 2$ in the last inequality at the expense of a stronger norm in the right-hand side and also drop the infimum in the left hand-side at the expense of imposing a boundary condition on $\mathbf{u}$. The idea that the exponent $1 / 2$ can be dropped is due to Blanchard [2].

The paper is organized as follows. In Section 2 we define the pure displacement problem in nonlinear elasticity and prove that its total energy possesses 
a unique global minimizer. This proof hinges on the nonlinear Korn inequality established in Section 3. Section 3 is independent of Section 2.

Vector and matrix fields are denoted by boldface letters. The gradient of a vector field $\mathbf{u}$ is the matrix field $\nabla \mathbf{u}$, whose columns are the partial derivatives of $\mathbf{u}$. The divergence of matrix field $\mathbf{A}$ is the vector field $\operatorname{div} \mathbf{A}$, whose components are the divergences of the row vectors of $\mathbf{A}$. The notations $L^{p}, W^{k, p}$ or $H^{k}$ if $p=2$, and $\mathcal{C}^{k}$ denote respectively Lebesgue spaces, Sobolev spaces, and $k$ times continuously differentiable functions. The Lebesque norms of vector and matrix fields such as $\mathbf{u} \in L^{\infty}\left(\Omega ; \mathbb{R}^{3}\right)$ and $\mathbf{E} \in L^{2}\left(\Omega ; \mathbb{S}^{3}\right)$ are denoted and defined by

$$
\|\mathbf{u}\|_{L^{\infty}(\Omega)}=\operatorname{ess-\operatorname {sup}}\{|\mathbf{u}(x)| ; x \in \Omega\} \text { and }\|\mathbf{E}\|_{L^{\infty}(\Omega)}=\left(\int_{\Omega}|\mathbf{E}(x)|^{2} d x\right)^{1 / 2},
$$

where $|\mathbf{u}(x)|$ and $|\mathbf{E}(x)|$ denote respectively the Euclidean norm of $\mathbf{u}(x) \in \mathbb{R}^{3}$ and the Frobenius norm of $\mathbf{E}(x) \in \mathbb{S}^{3}$.

The results of this paper have been announced in [6].

\section{The pure displacement problem in nonlinear elasticity: existence of a minimizer}

Consider a body with reference configuration $\Omega \subset \mathbb{R}^{3}$ subjected to dead body forces of density f : $\Omega \rightarrow \mathbb{R}^{3}$. Assume that the material constituting the body is hyperelastic and satisfies the axiom of frame indifference. This means that there exists a function $W: \bar{\Omega} \times \mathbb{S}^{3} \rightarrow \mathbb{R}$ such that the total energy associated with a deformation $\mathbf{u}: \Omega \rightarrow \mathbb{R}^{3}$ of the body is

$$
J(\mathbf{u})=\int_{\Omega} W(x, \mathbf{E}(\mathbf{u})(x)) d x-\int_{\Omega} \mathbf{f}(x) \cdot \mathbf{u}(x) d x
$$

where

$$
\mathbf{E}(\mathbf{u})=\frac{1}{2}\left(\nabla \mathbf{u}^{T} \nabla \mathbf{u}-\mathbf{I}\right)
$$

denotes the Green - Saint Venant strain tensor. The superscript ${ }^{T}$ to the right of a matrix (or matrix field) denotes the transpose of this matrix (or matrix field). The boldface letter $\mathbf{I}$ denotes the matrix field defined by $\mathbf{I}(x)=I$ for all $x \in \Omega$, where $I$ denotes the $3 \times 3$ identity matrix.

Assume that all admissible deformations $\mathbf{u}: \Omega \rightarrow \mathbb{R}^{3}$ preserve orientation, in the sense that

$$
\operatorname{det}(\nabla \mathbf{u}(x))>0 \text { for almost all } x \in \Omega \text {, }
$$

and satisfy the boundary condition of place

$$
\mathbf{u}(x)=x \text { for all } x \in \partial \Omega .
$$

Assume in addition that $\Omega, \mathbf{f}$, and $W$ satisfy the following assumptions: 
(a) The set $\Omega$ is bounded, connected, open, with boundary $\Gamma=\partial \Omega$ of class $\mathcal{C}^{2}$, the set $\Omega$ being locally on the same side of its boundary,

(b) $\mathbf{f} \in L^{p}\left(\Omega ; \mathbb{R}^{3}\right)$ for a certain $p>3$,

(c) The function $W: \bar{\Omega} \times \mathbb{S}^{3} \rightarrow \mathbb{R}$ is of class $\mathcal{C}^{3}$ and $\frac{\partial W}{\partial E}(x, 0)=0$ for all $x \in \bar{\Omega}$,

(d) There exists constants $\alpha>0$ and $\varepsilon>0$ such that, for all $x \in \bar{\Omega}, H \in \mathbb{S}^{3}$, $E \in \mathbb{S}^{3},|E|<\varepsilon$,

$$
W(x, E+H) \geq W(x, E)+\frac{\partial W}{\partial E}(x, E): H+\alpha|H|^{2} .
$$

The notations $\Sigma: H,|H|$, and $\frac{\partial W}{\partial E}$ denote respectively the inner product of two matrices, the Frobenius norm of a matrix, and the derivative of the function $W$ with respect to the its second variable. Specifically,

$$
\begin{aligned}
& \Sigma: H=\operatorname{tr}\left(\Sigma^{T} H\right), \quad|H|=\sqrt{H: H}, \\
& \frac{\partial W}{\partial E}(x, E): H=\lim _{t \rightarrow 0} \frac{W(x, E+t H)-W(x, E)}{t} .
\end{aligned}
$$

The assumption (c) means that $\Omega$ is a natural configuration of the body. The assumption (d) implies that $W(x, \cdot)$ is convex in a neighborhood of the zero matrix. But this does not imply that $J$ is convex, so the direct methods in the calculus of variations cannot be used to prove the existence of a minimizer.

In this setting, the pure displacement problem in nonlinear elasticity consists in minimizing the total energy

$$
\begin{aligned}
J(\mathbf{u})= & \int_{\Omega} W(x, \mathbf{E}(\mathbf{u})(x)) d x-\int_{\Omega} \mathbf{f}(x) \cdot \mathbf{u}(x) d x, \\
& \text { where } \mathbf{E}(\mathbf{u})=\frac{1}{2}\left(\nabla \mathbf{u}^{T} \nabla \mathbf{u}-\mathbf{I}\right),
\end{aligned}
$$

over the following set of admissible deformations

$$
\mathcal{M}=\left\{\mathbf{u} \in W^{1,4}\left(\Omega ; \mathbb{R}^{3}\right) ; \operatorname{det}(\nabla \mathbf{u})>0 \text { a.e. in } \Omega, \mathbf{u}(x)=x \text { for all } x \in \Gamma\right\} .
$$

Note that $J(\mathbf{u})$ is well defined in $\mathbb{R} \cup\{+\infty\}$. To see this, note first that $\mathbf{E}(\mathbf{u})=\frac{1}{2}\left(\nabla \mathbf{u}^{T} \nabla \mathbf{u}-\mathbf{I}\right) \in L^{2}\left(\Omega ; \mathbb{S}^{3}\right)$ since $\mathbf{u} \in W^{1,4}\left(\Omega ; \mathbb{R}^{3}\right)$ and that, thanks to assumption (d),

$$
W(x, \mathbf{E}(\mathbf{u})(x)) \geq W(x, 0)+\frac{\partial W}{\partial E}(x, 0): \mathbf{E}(\mathbf{u})(x)+\alpha|\mathbf{E}(\mathbf{u})(x)|^{2} .
$$

The function in the left-hand side is measurable and the function in the righthand side belongs to $L^{1}(\Omega)$, since $\Omega$ is bounded, $W$ is of class $\mathcal{C}^{3}$, and $\mathbf{E}(\mathbf{u}) \in$ $L^{2}\left(\Omega ; \mathbb{S}^{3}\right)$. Hence $W(x, \mathbf{E}(\mathbf{u})(x))$ is the sum of a function in $L^{1}(\Omega)$ and a measurable positive function. 
The next theorem asserts that the pure displacement problem in nonlinear elasticity has a unique solution provided the density of applied forces are small enough in $L^{p}(\Omega)$-norm.

Theorem 1 There exists a constant $\delta>0$ with the following property: Given any $\mathbf{f} \in L^{p}\left(\Omega ; \mathbb{R}^{3}\right)$ such that $\|\mathbf{f}\|_{L^{p}(\Omega)}<\delta$, there exists a unique deformation $\mathbf{v} \in \mathcal{M}$ such that

$$
J(\mathbf{v}) \leq J(\mathbf{u}) \text { for all } \mathbf{u} \in \mathcal{M} .
$$

Moreover, $\mathbf{v}$ belongs to the space $\mathcal{C}^{1}\left(\bar{\Omega} ; \mathbb{R}^{3}\right)$, is injective from $\bar{\Omega}$ into $\mathbb{R}^{3}$, and satisfies $\operatorname{det} \nabla \mathbf{v}(x)>0$ for all $x \in \bar{\Omega}$.

Proof. First we prove the existence of solution to the Euler-Lagrange equation formally derived from the total energy $J$, namely

$$
-\operatorname{div}\left(\nabla \mathbf{u} \frac{\partial W}{\partial E}(\cdot, \mathbf{E}(\mathbf{u}))\right)=\mathbf{f} \text { in } \Omega .
$$

Note that this equation and the boundary condition $\mathbf{u}(x)=x$ for all $x \in \Gamma$ constitute the boundary value problem of nonlinear elasticity.

We show that this problem has a solution in $W^{2, p}\left(\Omega ; \mathbb{R}^{3}\right)$ by applying the implicit function theorem to the mapping $\mathcal{A}: X \rightarrow Y$ defined by

$$
\begin{gathered}
\mathcal{A}(\mathbf{u})=-\operatorname{div}\left(\nabla \mathbf{u} \frac{\partial W}{\partial E}(\cdot, \mathbf{E}(\mathbf{u}))\right), \\
X=\left\{\mathbf{u} \in W^{2, p}\left(\Omega ; \mathbb{R}^{3}\right) ; \mathbf{u}(x)=x \text { for all } x \in \Gamma\right\}, Y=L^{p}\left(\Omega ; \mathbb{R}^{3}\right) .
\end{gathered}
$$

Note that $\mathcal{A}(\mathbf{i d})=\mathbf{0}$, where id $\in X$ and $\mathbf{0} \in Y$ are defined by $\mathbf{i d}(x)=x$ and $\mathbf{0}(x)=0$ for all $x \in \Omega$.

Since $W^{1, p}\left(\Omega ; \mathbb{S}^{3}\right)$ is an algebra, the mapping $\mathcal{A}$ is well defined, is of class $\mathcal{C}^{1}$, and its tangent map at id is the linear map $\mathcal{T}: X_{0} \rightarrow Y$ defined by

$$
\begin{gathered}
\mathcal{T}(\mathbf{d})=-\operatorname{div}\left(\frac{\partial^{2} W}{\partial E^{2}}(\cdot, 0)\left(\nabla_{s} \mathbf{d}\right)\right), \quad \text { where } \nabla_{s} \mathbf{d}=\frac{\nabla \mathbf{d}^{T}+\nabla \mathbf{d}}{2}, \\
X_{0}=\left\{\mathbf{d} \in W^{2, p}\left(\Omega ; \mathbb{R}^{3}\right) ; \mathbf{d}(x)=0 \text { for all } x \in \Gamma\right\},
\end{gathered}
$$

where, for all $H \in \mathbb{S}^{3}$,

$$
\frac{\partial^{2} W}{\partial E^{2}}(x, 0)(H):=\lim _{t \rightarrow 0} \frac{1}{t}\left(\frac{\partial W}{\partial E}(x, t H)-\frac{\partial W}{\partial E}(x, 0)\right) .
$$

Since $\frac{\partial^{2} W}{\partial E^{2}}(x, H) \geq 2 \alpha|H|^{2}$ for all $x \in \bar{\Omega}$ and for all $H \in \mathbb{S}^{3}$ (assumption (d)), we have

$$
\int_{\Omega} \frac{\partial^{2} W}{\partial E^{2}}(\cdot, 0)\left(\nabla_{s} \mathbf{d}\right): \nabla \mathbf{d} d x=\int_{\Omega} \frac{\partial^{2} W}{\partial E^{2}}(\cdot, 0)\left(\nabla_{s} \mathbf{d}\right): \nabla_{s} \mathbf{d} d x \geq 2 \alpha\left\|\nabla_{s} \mathbf{d}\right\|_{L^{2}(\Omega)}^{2} .
$$

By Korn's inequality, there exists a constant $C>0$ such that, for all $\mathbf{d} \in X_{0}$,

$$
\int_{\Omega} \frac{\partial^{2} W}{\partial E^{2}}(\cdot, 0)\left(\nabla_{s} \mathbf{d}\right): \nabla \mathbf{d} d x \geq C\|\mathbf{d}\|_{H^{1}(\Omega)}^{2} .
$$


This inequality and the regularity assumptions on $W$ and $\Omega$ imply that the linear map $\mathcal{T}$ is an isomorphism from $X_{0}$ onto $Y$; cf., e.g., Quintela-Estevez [7, Theorem 4.5]. Note that the inequality above insures the existence of a solution in $H^{1}\left(\Omega ; \mathbb{R}^{3}\right)$ to the variational equation associated with the equation $\mathcal{T}(\mathbf{d})=\mathbf{f}$ (which is in effect an elliptic system of linear partial differential equations) and that the regularity assumptions on $W, \Omega$, and $\mathbf{f}$, insures that this solution is of class $W^{2, p}\left(\Omega ; \mathbb{R}^{3}\right)$.

The hypotheses of the implicit function theorem being thus satisfied, there exists two constants $\delta>0$ and $\varepsilon_{1}>0$ such that, for each $\mathbf{f} \in Y$ such that $\|\mathbf{f}\|_{L^{p}(\Omega)}<\delta$, there exists a unique element $\mathbf{v}=\mathbf{v}(\mathbf{f}) \in X$ that satisfies $\mathcal{A}(\mathbf{v})=\mathbf{f}$ and $\|\mathbf{v}-\mathbf{i d}\|_{W^{2, p}(\Omega)}<\varepsilon_{1}$. Since the implicit function $\mathbf{f} \mapsto \mathbf{v}(\mathbf{f})$ is continous and the Sobolev embedding $W^{2, p}(\Omega) \subset \mathcal{C}^{1}(\bar{\Omega})$ is also continuous, we have

$$
\lambda(\delta):=\sup _{\|\mathbf{f}\|_{L^{p}(\Omega)}<\delta}\|\nabla(\mathbf{v}(\mathbf{f})-\mathbf{i d})\|_{L^{\infty}(\Omega)} \rightarrow 0 \text { as } \delta \rightarrow 0 .
$$

By choosing $\delta$ such that $\lambda(\delta)<1$, we deduce that $\operatorname{det}(\nabla \mathbf{v}(x))>0$ for all $x \in \bar{\Omega}$; cf. Ciarlet [3, Theorem 5.5-1]. This in turn implies that $\mathbf{v}$ is injective from $\bar{\Omega}$ into $\mathbb{R}^{3}$, since $\mathbf{v}(x)=x$ for all $x \in \Gamma$; cf. Ciarlet [3, Theorem 5.5-2].

Now we prove that $\mathbf{v}$ is the unique minimizer of the functional $J$ over $\mathcal{M}$ provided that $\delta$ is chosen small enough. Choose $\delta$ such that $\lambda(\delta)<\varepsilon / 3$ (the constant $\varepsilon$ is that appearing in assumption (d)). Then

$$
\|\mathbf{E}(\mathbf{v})\|_{L^{\infty}(\Omega)} \leq 3\|\nabla(\mathbf{v}-\mathbf{i d})\|_{L^{\infty}(\Omega)}<3 \lambda(\delta)<\varepsilon .
$$

Let $\mathbf{u} \in \mathcal{M}$ and set $\mathbf{w}=\mathbf{u}-\mathbf{v}$. Note that $\mathbf{w} \in W^{1,4}\left(\Omega ; \mathbb{R}^{3}\right)$ and that its trace on the boundary $\Gamma$ of $\Omega$ satisfies $\mathbf{w}(x)=0$ for almost all $x \in \Gamma$. By assumption (d), we have on the one hand that

$$
\begin{aligned}
J(\mathbf{u})-J(\mathbf{v}) & =\int_{\Omega}(W(\cdot, \mathbf{E}(\mathbf{u}))-W(\cdot, \mathbf{E}(\mathbf{v}))) d x-\int_{\Omega} \mathbf{f} \cdot \mathbf{w} d x \\
& \geq \int_{\Omega}\left(\frac{\partial W}{\partial E}(\cdot, \mathbf{E}(\mathbf{v})):(\mathbf{E}(\mathbf{u})-\mathbf{E}(\mathbf{v}))+\alpha|\mathbf{E}(\mathbf{u})-\mathbf{E}(\mathbf{v})|^{2}\right) d x-\int_{\Omega} \mathbf{f} \cdot \mathbf{w} d x .
\end{aligned}
$$

On the other hand, since $\frac{\partial W}{\partial E}(x, \mathbf{E}(\mathbf{v})(x)) \in \mathbb{S}^{3}$ for all $x \in \bar{\Omega}$, we also have

$$
\begin{aligned}
\frac{\partial W}{\partial E}(\cdot, \mathbf{E}(\mathbf{v})):(\mathbf{E}(\mathbf{u})-\mathbf{E}(\mathbf{v})) & =\frac{\partial W}{\partial E}(\cdot, \mathbf{E}(\mathbf{v})):\left(\frac{\nabla \mathbf{v}^{T} \nabla \mathbf{w}+\nabla \mathbf{w}^{T} \nabla \mathbf{v}}{2}+\frac{\nabla \mathbf{w}^{T} \nabla \mathbf{w}}{2}\right) \\
& =\frac{\partial W}{\partial E}(\cdot, \mathbf{E}(\mathbf{v})): \nabla \mathbf{v}^{T} \nabla \mathbf{w}+\frac{\partial W}{\partial E}(\cdot, \mathbf{E}(\mathbf{v})): \frac{\nabla \mathbf{w}^{T} \nabla \mathbf{w}}{2}
\end{aligned}
$$

and, since the trace of the vector field $\mathbf{w}$ on the boundary of $\Omega$ satisfies $\mathbf{w}(x)=0$

for almost all $x \in \Gamma$,

$$
\begin{aligned}
\int_{\Omega} \frac{\partial W}{\partial E}(\cdot, \mathbf{E}(\mathbf{v})): \nabla \mathbf{v}^{T} \nabla \mathbf{w} d x & =\int_{\Omega} \nabla \mathbf{v} \frac{\partial W}{\partial E}(\cdot, \mathbf{E}(\mathbf{v})): \nabla \mathbf{w} d x \\
& =-\int_{\Omega} \operatorname{div}\left(\nabla \mathbf{v} \frac{\partial W}{\partial E}(\cdot, \mathbf{E}(\mathbf{v}))\right) \cdot \mathbf{w} d x \\
& =\int_{\Omega} \mathcal{A}(\mathbf{v}) \cdot \mathbf{w} d x
\end{aligned}
$$


Hence

$$
\begin{array}{r}
J(\mathbf{u})-J(\mathbf{v}) \geq \int_{\Omega}(\mathcal{A}(\mathbf{v})-\mathbf{f}) \cdot \mathbf{w} d x+\int_{\Omega} \frac{\partial W}{\partial E}(\cdot, \mathbf{E}(\mathbf{v})): \frac{\nabla \mathbf{w}^{T} \nabla \mathbf{w}}{2} d x \\
+\alpha \int_{\Omega}|\mathbf{E}(\mathbf{u})-\mathbf{E}(\mathbf{v})|^{2} d x .
\end{array}
$$

Since $\mathcal{A}(\mathbf{v})=\mathbf{f}$, it follows that

$$
J(\mathbf{u})-J(\mathbf{v}) \geq \frac{\alpha}{4}\left\|\nabla \mathbf{u}^{T} \nabla \mathbf{u}-\nabla \mathbf{v}^{T} \nabla \mathbf{v}\right\|_{L^{2}(\Omega)}^{2}-\beta(\delta)\|\nabla \mathbf{w}\|_{L^{2}(\Omega)}^{2},
$$

where $\beta(\delta)=\frac{1}{2}\left\|\frac{\partial W}{\partial E}(\cdot, \mathbf{E}(\mathbf{v}))\right\|_{L^{\infty}(\Omega)}$. But the nonlinear Korn inequality proven independently in the next section (Theorem 2) shows that

$$
\|\nabla \mathbf{w}\|_{L^{2}(\Omega)} \leq \frac{C}{(1-\lambda(\delta))^{7 / 2}}\left\|\nabla \mathbf{u}^{T} \nabla \mathbf{u}-\nabla \mathbf{v}^{T} \nabla \mathbf{v}\right\|_{L^{2}(\Omega)}
$$

for some universal constant $C$. Therefore

$$
J(\mathbf{u})-J(\mathbf{v}) \geq\left(\frac{\alpha(1-\lambda(\delta))^{7}}{4 C^{2}}-\beta(\delta)\right)\|\nabla \mathbf{w}\|_{L^{2}(\Omega)}^{2} .
$$

It remains to prove that $\frac{\alpha(1-\lambda(\delta))^{7}}{4 C^{2}}-\beta(\delta)>0$ if the constant $\delta$ given by the implicit function theorem is chosen small enough. Since $\|\mathbf{E}(\mathbf{v})\|_{L^{\infty}(\Omega)} \leq$ $3\|\nabla(\mathbf{v}-\mathbf{i d})\|_{L^{\infty}(\Omega)}<3 \lambda(\delta)$, we have

$$
\beta(\delta) \leq \sup _{(x, E) \in K} \frac{1}{2}\left|\frac{\partial W}{\partial E}(x, E)\right|, \text { where } K=\{(x, E) ; x \in \bar{\Omega},|E|<3 \lambda(\delta)\} .
$$

But $\frac{\partial W}{\partial E}$ is uniformly continuous on $K$ and $\frac{\partial W}{\partial E}(x, 0)=0$ for all $x \in \bar{\Omega}$ by assumption (c). Therefore $\beta(\delta) \rightarrow 0$ as $\lambda(\delta) \rightarrow 0$. Since $\lambda(\delta) \rightarrow 0$ and $\frac{\alpha(1-\lambda(\delta))^{7}}{4 C^{2}} \rightarrow \frac{\alpha}{4 C^{2}}>0$ as $\delta \rightarrow 0$, it suffices to choose $\delta$ small enough.

That the vector field $\mathbf{v}$ is the unique minimizer of the total energy $J$ over the set of all admissible deformations $\mathcal{M}$ is a simple consequence of the inequality

$$
J(\mathbf{u})-J(\mathbf{v}) \geq\left(\frac{\alpha(1-\lambda(\delta))^{7}}{4 C^{2}}-\beta(\delta)\right)\|\nabla \mathbf{w}\|_{L^{2}(\Omega)}^{2}>0
$$

for all $\mathbf{u} \in \mathcal{M}$ such that $\mathbf{u} \neq \mathbf{v}$.

Remark. Theorem 1 still holds true if $W$ is of class $\mathcal{C}^{3}$ only over a neighborhood of $\bar{\Omega} \times\{0\}$ in $\bar{\Omega} \times \mathbb{S}^{3}$. For instance, it suffices to assume that $W$ is of class $\mathcal{C}^{3}$ over $\bar{\Omega} \times\left\{E \in \mathbb{S}^{3} ;|E|<\varepsilon\right\}$, for some $\varepsilon>0$.

\section{A nonlinear Korn inequality with boundary conditions}

The objective of this section is to prove the nonlinear Korn inequality used in the proof of Theorem 1. Note that another nonlinear Korn inequality has been 
established earlier by Ciarlet \& Mardare [4, Lemma 6], but this inequality is not useful here. Note also that the constant appearing in the next theorem does not depend on $\mathbf{v}$, which has been essential in the proof of Theorem 1 .

Theorem 2 Let $\Omega$ be a bounded connected open subset of $\mathbb{R}^{3}$ with a Lipschitzcontinuous boundary $\Gamma=\partial \Omega$, the set $\Omega$ being locally on the same side of its boundary. Let $\lambda<1$ and let $\mathbf{v} \in \mathcal{C}^{1}\left(\bar{\Omega} ; \mathbb{R}^{3}\right)$ be an injective mapping satisfying $\|\nabla(\mathbf{v}-\mathbf{i d})\|_{L^{\infty}(\Omega)} \leq \lambda$. Then there exists a constant $C$ independent of $\mathbf{v}$ such that

$$
\|\nabla \mathbf{u}-\nabla \mathbf{v}\|_{L^{2}(\Omega)} \leq \frac{C}{(1-\lambda)^{7 / 2}}\left\|\nabla \mathbf{u}^{T} \nabla \mathbf{u}-\nabla \mathbf{v}^{T} \nabla \mathbf{v}\right\|_{L^{2}(\Omega)}
$$

for all $\mathbf{u} \in W^{1,4}\left(\bar{\Omega} ; \mathbb{R}^{3}\right)$ that satisfies $\operatorname{det}(\nabla \mathbf{u}(x))>0$ for almost all $x \in \Omega$ and $\mathbf{u}(x)=\mathbf{v}(x)$ for almost all $x \in \Gamma$.

Proof. Let $B \subset \mathbb{R}^{3}$ be an open ball containing $\bar{\Omega}$. Extend the mappings $\mathbf{v}$ and $\mathbf{u}$ to $\bar{B}$ such that $\mathbf{v}(x)=x$ for all $x \in \partial B, \mathbf{u}(x)=x$ for all $x \in \bar{B} \backslash \bar{\Omega}$ (the extensions are denoted by the same letters), and

$$
\begin{array}{ll}
\mathbf{v} \in \mathcal{C}^{1}\left(\bar{B} ; \mathbb{R}^{3}\right), & |\nabla \mathbf{v}(x)-I| \leq \lambda<1 \text { for all } x \in \bar{B} \\
\mathbf{u} \in W^{1,4}\left(B ; \mathbb{R}^{3}\right), & \operatorname{det}(\nabla \mathbf{u}(x))>0 \text { for almost all } x \in B .
\end{array}
$$

The relations $|\nabla \mathbf{v}(x)-I|<1$ for all $x \in \bar{B}$ and $\mathbf{v}(x)=x$ for all $x \in \partial B$ imply that $\operatorname{det}(\nabla \mathbf{v}(x))>0$ for all $x \in \bar{B}$, that $\mathbf{v}$ is injective from $\bar{B}$ into $\mathbb{R}^{3}$, and that $\mathbf{v}(B)=B$ and $\mathbf{v}(\bar{B})=\bar{B}$; cf. Ciarlet [3, Theorems 5.5.1 and 5.5-2].

Since $|\nabla \mathbf{v}(x)-I| \leq \lambda<1$ for all $x \in \bar{B}$, the inverse mapping $\mathbf{v}^{-1}: \bar{B} \rightarrow \bar{B}$ belongs the space $\mathcal{C}^{1}\left(\bar{B} ; \mathbb{R}^{3}\right)$ and, for all $x \in \bar{B},\left|\nabla \mathbf{v}^{-1}(x)-I\right| \leq \frac{\lambda}{1-\lambda}$. Define the composite mapping $\varphi=\mathbf{u} \circ \mathbf{v}^{-1}: B \rightarrow \mathbb{R}^{3}$ and note that $\varphi \in W^{1,4}\left(B ; \mathbb{R}^{3}\right)$. Then the geometric rigidity lemma of Friesecke, James \& Müller [5, Theorem 3.1] implies on the one hand that

$$
\inf _{R \in \mathbb{O}_{+}^{3}} \int_{B}|\nabla \varphi(x)-R|^{2} d x \leq K \int_{B} \inf _{Q \in \mathbb{O}_{+}^{3}}|\nabla \varphi(x)-Q|^{2} d x
$$

for some constant $K$ independent of $\mathbf{u}$ and $\mathbf{v}$.

On the other hand, since $\varphi(x)=x$ for almost all $x \in B \backslash \Omega$, the $3 \times 3$ identity matrix $I$ satisfies $I=\frac{1}{|B \backslash \Omega|} \int_{B \backslash \Omega} \nabla \varphi(x) d x$, where $|B \backslash \Omega|$ denotes the Lebesque measure $d x$ of set $B \backslash \Omega$. Then, for every $R \in \mathbb{O}_{+}^{3}$, we have

$$
\begin{aligned}
\|\nabla \boldsymbol{\varphi}-\mathbf{I}\|_{L^{2}(B)} & \leq\left(\int_{B}|\nabla \boldsymbol{\varphi}(x)-R|^{2} d x\right)^{1 / 2}+|B|^{1 / 2}|R-I| \\
& \leq\left(\int_{B}|\nabla \boldsymbol{\varphi}(x)-R|^{2} d x\right)^{1 / 2}+\frac{|B|^{1 / 2}}{|B \backslash \Omega|} \int_{B \backslash \Omega}|R-\nabla \boldsymbol{\varphi}(x)| d x \\
& \leq\left(1+\frac{|B|^{1 / 2}}{|B \backslash \Omega|^{1 / 2}}\right)\left(\int_{B}|\nabla \boldsymbol{\varphi}(x)-R|^{2} d x\right)^{1 / 2}
\end{aligned}
$$


Hence

$$
\|\nabla \boldsymbol{\varphi}-\mathbf{I}\|_{L^{2}(B)} \leq C_{0}\left(\int_{B} \inf _{Q \in \mathbb{O}_{+}^{3}}|\nabla \varphi(x)-Q|^{2} d x\right)^{1 / 2}
$$

where $C_{0}=K^{1 / 2}\left(1+\frac{|B|^{1 / 2}}{|B \backslash \Omega|^{1 / 2}}\right)$.

To compute the infimum in the right-hand side of the above inequality, let $F$ be any $3 \times 3$ matrix with $\operatorname{det}(F)>0$. Let $F=R U$, where $R \in \mathbb{O}_{+}^{3}$ and $U \in \mathbb{S}_{>}^{3}$, be the polar decomposition of $F$ and let $\lambda_{1} \geq \lambda_{2} \geq \lambda_{3}>0$ be the eigenvalues of the symmetric positive-definite matrix $U$. Let $\Lambda$ denote the diagonal matrix with $\lambda_{i}$ on its diagonal and let $P \in \mathbb{O}_{+}^{3}$ such that $U=P^{T} \Lambda P$. Then

$$
\begin{aligned}
\inf _{Q \in \mathbb{O}_{+}^{3}}|F-Q|^{2} & =|\Lambda|^{2}+|I|^{2}-2 \sup _{Q \in \mathbb{O}_{+}^{3}} \Lambda:\left(P R^{T} Q P^{T}\right) \\
& =|\Lambda|^{2}+|I|^{2}-2 \sup _{Q^{\prime} \in \mathbb{O}_{+}^{3}} \Lambda: Q^{\prime} .
\end{aligned}
$$

Since the column vectors of an orthogonal matrix are of norm $=1$, this implies that

$$
\begin{aligned}
\inf _{Q \in \mathbb{O}_{+}^{3}}|F-Q|^{2} & =|\Lambda|^{2}+|I|^{2}-2 \Lambda: I=|\Lambda-I|^{2}=\sum_{i=1}^{3}\left(\lambda_{i}-1\right)^{2} \\
& \leq \sum_{i=1}^{3}\left(\lambda_{i}^{2}-1\right)^{2}=\left|\Lambda^{2}-I^{2}\right|^{2}=\left|U^{2}-I\right|^{2}=\left|F^{T} F-I\right|^{2} .
\end{aligned}
$$

Using this inequality in the right-hand side of the previous inequality we deduce that

$$
\|\nabla \boldsymbol{\varphi}-\mathbf{I}\|_{L^{2}(B)} \leq C_{0}\left\|\nabla \boldsymbol{\varphi}^{T} \nabla \boldsymbol{\varphi}-\mathbf{I}\right\|_{L^{2}(B)},
$$

or equivalently (recall that $\varphi(x)=x$ for almost all $x \in B \backslash \Omega$ )

$$
\|\nabla \varphi-\mathbf{I}\|_{L^{2}(\Omega)} \leq C_{0}\left\|\nabla \varphi^{T} \nabla \varphi-\mathbf{I}\right\|_{L^{2}(\Omega)}
$$

Now we perform the change of variables $x=\mathbf{v}(y)$ in the integrals appearing in the above inequality. Since $\boldsymbol{\varphi}(x)=\mathbf{u}\left(\mathbf{v}^{-1}(x)\right)$ and $\nabla \boldsymbol{\varphi}(\mathbf{v}(y))=$ $\nabla \mathbf{u}(y)(\nabla \mathbf{v}(y))^{-1}$ for almost all $y \in \Omega$, we deduce that

$$
\int_{\Omega}\left|\nabla \mathbf{u}(\nabla \mathbf{v})^{-1}-\mathbf{I}\right|^{2} \operatorname{det}(\nabla \mathbf{v}) d y \leq C_{0}^{2} \int_{\Omega}\left|(\nabla \mathbf{v})^{-T} \nabla \mathbf{u}^{T} \nabla \mathbf{u}(\nabla \mathbf{v})^{-1}-\mathbf{I}\right|^{2} \operatorname{det}(\nabla \mathbf{v}) d y
$$

which next implies that

$$
\int_{\Omega}|\nabla \mathbf{u}-\nabla \mathbf{v}|^{2} \frac{\operatorname{det}(\nabla \mathbf{v})}{|\nabla \mathbf{v}|^{2}} d y \leq C_{0}^{2} \int_{\Omega}\left|\nabla \mathbf{u}^{T} \nabla \mathbf{u}-\nabla \mathbf{v}^{T} \nabla \mathbf{v}\right|^{2}\left|(\nabla \mathbf{v})^{-1}\right|^{4} \operatorname{det}(\nabla \mathbf{v}) d y
$$

Since $|\nabla \mathbf{v}(y)-I| \leq \lambda<1$ and $\left|\nabla \mathbf{v}^{-1}(y)-I\right| \leq \frac{\lambda}{1-\lambda}$ for all $y \in \bar{\Omega}$, we deduce that the singular values of the matrix $\nabla \mathbf{v}(y)$ are contained in the interval 
$\left[\frac{1-\lambda}{\lambda+(1-\lambda)|I|},|I|+\lambda\right]$. Therefore there exists constants $C_{1}$ and $C_{2}$ such that, at every point of $\bar{\Omega}$,

$$
\frac{\operatorname{det}(\nabla \mathbf{v})}{|\nabla \mathbf{v}|^{2}} \geq C_{1}(1-\lambda)^{3} \text { and }\left|(\nabla \mathbf{v})^{-1}\right|^{4} \operatorname{det}(\nabla \mathbf{v}) \leq \frac{C_{2}}{(1-\lambda)^{4}}
$$

We then infer from the previous inequality that

$$
\|\nabla \mathbf{u}-\nabla \mathbf{v}\|_{L^{2}(\Omega)} \leq \frac{C}{(1-\lambda)^{7 / 2}}\left\|\nabla \mathbf{u}^{T} \nabla \mathbf{u}-\nabla \mathbf{v}^{T} \nabla \mathbf{v}\right\|_{L^{2}(\Omega)},
$$

where $C=C_{0}\left(C_{2} / C_{1}\right)^{1 / 2}$.

Remark. The nonlinear Korn inequality of Theorem 2 remains valid in any dimension and for deformation fields that are possibly non injective and satisfy more general boundary conditions, as will be shown elsewhere.

Acknowledgments. The author is grateful to the organizers of the conference celebrating 100 years from the foundation of the Alexandru Myller Mathematical Seminar of the University of Iaşi for their hospitality.

\section{References}

[1] J. Ball, Convexity conditions and existence theorems in nonlinear elasticity, Arch. Rational Mech. Anal. 63, 337-403 (1977).

[2] D. Blanchard, Personal communication.

[3] P.G. Ciarlet, Mathematical Elasticity, Volume I: Three-Dimensional Elasticity, North-Holland, Amsterdam, 1988.

[4] P.G. Ciarlet and C. Mardare, Continuity of a deformation in $H^{1}$ as a function of its Cauchy-Green tensor in $L^{1}$, J. of Nonlinear Science 14, 415-427 (2004).

[5] G. Friesecke, R.D. James, and S. Müller, A theorem on geometric rigidity and the derivation of nonlinear plate theory from three dimensional elasticity, Comm. Pure Appl. Math. 55, 1461-1506 (2002).

[6] C. Mardare, A nonlinear Korn inequality with boundary conditions and its relation to the existence of minimizers in nonlinear elasticity, C. R. Acad. Sci. Paris, Ser. I, submitted.

[7] P. Quintela-Estevez, Critical points in the energy of hyperelastic materials, RAIRO Model. Math. Anal. Numer. 24, 103132 (1990).

[8] K. Zhang, Energy minimizers in nonlinear elastostatics and the implicit function theorem, Arch. Rational Mech. Anal. 114, 95-117 (1991). 